\title{
Hamilton-Jacobi Approach for Power-Law Potentials
}

\author{
R. C. Santos ${ }^{1}$, J. Santos ${ }^{1}$, J. A. S. Lima ${ }^{2}$ \\ ${ }^{1}$ Departamento de Física, UFRN, 59072-970, Natal, RN, Brazil \\ ${ }^{2}$ Instituto de Astronomia, Geofísica e Ciências Atmosféricas - USP \\ Rua do Matão, 1226 - Cid. Universitária, 05508-900, São Paulo, SP, Brazil
}

Received on 25 August, 2006

\begin{abstract}
The classical and relativistic Hamilton-Jacobi approach is applied to the one-dimensional homogeneous potential, $V(q)=\alpha q^{n}$, where $\alpha$ and $n$ are continuously varying parameters. In the non-relativistic case, the exact analytical solution is determined in terms of $\alpha, n$ and the total energy $E$. It is also shown that the non-linear equation of motion can be linearized by constructing a hypergeometric differential equation for the inverse problem $t(q)$. A variable transformation reducing the general problem to that one of a particle subjected to a linear force is also established. For any value of $n$, it leads to a simple harmonic oscillator if $E>0$, an "anti-oscillator" if $E<0$, or a free particle if $E=0$. However, such a reduction is not possible in the relativistic case. For a bounded relativistic motion, the first order correction to the period is determined for any value of $n$. For $n>>1$, it is found that the correction is just twice that one deduced for the simple harmonic oscillator $(n=2)$, and does not depend on the specific value of $n$.
\end{abstract}

Keywords: Hamilton-Jacobi equation; Power-law potentials

\section{INTRODUCTION}

The Hamilton-Jacobi (HJ) equation is a powerful method either to the relativistic and classical framework, and also plays a prominent role in quantum mechanics as a special route for a comprehension of the Schrödinger equation[1, 2]. The associated action variable method, which is a slight modification of the HJ approach, is also quite useful for determining frequencies and energies of periodic systems. Even for the general relativity theory, the importance of the HJ approach has long been recognized by many authors (see, for instance, [3] and Refs. therein). Indeed, one of the most elegant methods for describing geodesics and orbits of test particles in Schwarzchild and Kerr spacetimes, as well as for any stationary gravitational configuration, is provided by the relativistic HJ equation[3-5].

In the nonrelativistic domain, the standard applications of the HJ theory are the harmonic oscillator, the Kepler problem, and charged particles moving in electro-magnetic fields. Recently, the method has also been applied to the rocket problem[6]. Pars[7] studied the motion of a classical particle in a plane under central attraction derived from the potential, $V(r)=\alpha r^{n}$, for some particular values of $n$. However, to the best of our knowledge, if $\alpha$ and $n$ are continuously varying parameters the general solution has not been obtained even for the one-dimensional case.

In this letter we discuss an analytical solution for the power law potential both for the classical and relativistic case. As we shall see, the HJ equation for a particle moving under the action of a one-dimensional potential, $V(q)=\alpha q^{n}$, has a general and unified solution in terms of hypergeometric functions. The period of the motion as a function of the power index $n$ and of the $\alpha$ parameter are easily determined from the general solution. For completeness, we also show that such a problem may also be exactly solved starting from the nonlinear equation of motion by employing a slightly modified EulerLagrange approach. By changing the coordinate and adopting an auxiliary time, we show that the motion of the particle in such a potential may be reduced to the problem of a particle under the action of a linear force: an oscillator if $E>0$, an "anti-oscillator" if $E<0$ or a free-particle if $E=0$, where $E$ is the total energy of the particle. Classically, these results hold regardless of the value of $n$. Such a reduction cannot be implemented in the relativistic case. However, the general problem may be reduced to an integral, which is a natural extension of the earlier Synge's treatment for the relativistic harmonic oscillator[8].

\section{HAMILTON-JACOBI APPROACH}

The classical Lagrangian for a particle subject to the onedimensional potential, $V(q)=\alpha q^{n}$, reads

$$
L(q, \dot{q}, t)=\frac{1}{2} m \dot{q}^{2}-\alpha q^{n},
$$

where an overdot means total derivative. The parameters $\alpha$, $n$, vary continuously, and $m, q$ are, respectively, the mass of the particle and its generalized coordinate. The Hamiltonian is given by ( $p$ is the canonical momentum)

$$
H(q, p)=\frac{p^{2}}{2 m}+\alpha q^{n}=E
$$

and since it does not depend explicitly on the time, it represents a conserved quantity which is the energy of the system. The Hamilton-Jacobi equation for the Hamiltonian (2) assume the following form [1]

$$
\frac{1}{2 m}\left(\frac{\partial S}{\partial q}\right)^{2}+\alpha q^{n}+\frac{\partial S}{\partial t}=0
$$

where $S$ is the Hamilton's principal function. Since the explicit dependence of $S$ on time is involved only in the last term, 
the variables can be separated. Following standard lines, the solution is assumed to have the form

$$
S(q, E, t)=W(q, E)-E t,
$$

where $E$, the constant of integration, has been identified with the total energy. With this choice, the time is readily eliminated from (3), and Hamilton's principal function $S$ becomes

$$
S=\int \sqrt{2 m E-2 m \alpha q^{n}} d q-E t .
$$

It should be noticed that if the energy $E$ is positive, the constant $\alpha$ appearing in the potential may assume either negative or positive values, however, if $E \leq 0$, the allowed values of $\alpha$ are necessarily negative. Since $H=E$, such considerations also follows naturally from the positivity of the kinetic energy

$$
K=\frac{1}{2} m \dot{q}^{2}=E-\alpha q^{n} .
$$

The second integration constant is a consequence of the Jacobi transformation equation

$$
\beta=\frac{\partial S}{\partial E}=\sqrt{\frac{m}{2|E|}} \int_{0}^{q} \frac{d q}{\sqrt{1-\frac{\alpha}{E} q^{n}}}-t .
$$

The root of $|E|$ arises naturally if one takes for negative values of $E, E^{*}=-E$, and compute the partial derivative with respect to $E$. In order to integrate (7) we make the change of variable $u=(\alpha / E) q^{n}$. Thus we have that

$$
t+\beta=\sqrt{\frac{m}{2 n^{2}|E|}}\left(\frac{E}{\alpha}\right)^{1 / n} \int_{0}^{u} \frac{y^{1 / n-1} d y}{\sqrt{1-y}} .
$$

It is worth noticing that the integral appearing on the right hand side of the above expression is the incomplete Beta function[9]

$$
B_{u}(a, b)=\int_{0}^{u} y^{a-1}(1-y)^{b-1} d y,
$$

for $a=1 / n$ and $b=1 / 2$. Without loss of generality we may take $q(t=0)=0$, so that $\beta=0$ in (8). When the motion is finite, the particle moves back and forth (oscillatory motion), and the period $T$ can be easily determined. We see from (6) that at the turning points $(\dot{q}=0)$ the amplitude of the oscillation is $A=(E / \alpha)^{1 / n}$, and, therefore, $u_{\max }=1$, is the maximum value in the upper limit of the integral (8). The period of the motion is given by $T=4 t_{\max }$ and, taking these considerations into account, we find that equation (8) yields

$$
T=2 \sqrt{\frac{2 m}{\alpha n^{2}}} A^{1-1 / n} B(1 / n, 1 / 2),
$$

where $B(1 / n, 1 / 2)=\sqrt{\pi} \Gamma\left(\frac{1}{n}\right) / \Gamma\left(\frac{1}{n}+\frac{1}{2}\right)$ is the complete Beta function. The period of this oscillator is clearly dependent on the amplitude $A$, unless $n=2$. As should be expected, for $n=2$ equation (9) reduces to

$$
T=2 \pi \sqrt{\frac{m}{2 \alpha}},
$$

which is the well known result for the simple harmonic oscillator.

It should be recalled that the incomplete Beta function is related to the hypergeometric Gaussian $F(a, b ; c ; u)$ by the following identity: $B_{u}(a, b)=a^{-1} u^{a} F(a, 1-b ; 1+a ; u)$. It thus follows that the result (8) may be expressed as

$$
t+\beta=\sqrt{\frac{m}{2|E|}} q F\left(\frac{1}{2}, \frac{1}{n} ; \frac{1}{n}+1 ; \frac{\alpha}{E} q^{n}\right) .
$$

As a check, we notice that for $n=2$ the above expression can be written as

$$
\omega(t+\beta)=z F\left(\frac{1}{2}, \frac{1}{2} ; \frac{3}{2} ; z^{2}\right),
$$

where we have defined the variable $z=\sqrt{\alpha / E} q$ and $\omega=$ $2 \pi / T$ is the frequency of the oscillatory motion. Since $F\left(1 / 2,1 / 2 ; 3 / 2 ; z^{2}\right)=z^{-1} \arcsin z$ [9]; by returning to the old variable $q$, the above equation can be recast as

$$
q(t)=\sqrt{\frac{2 E}{m \omega^{2}}} \sin \omega(t+\beta),
$$

which describes the motion of a simple harmonic oscillator [1].

\section{MODIFIED EULER-LAGRANGE APPROACH}

We present here another method of solution for the problem outlined in the introduction. In principle, by considering that the $\mathrm{HJ}$ approach leads to the hypergeometric function for $t(q)$ as given by (10), it should be possible to obtain the differential hypergeometric equation starting directly from the equation of motion. The importance of this method is two-fold: the initial conditions remains arbitrary and the linearization procedure is a guarantee that we get the complete solution for the nonlinear differential equation governing the motion of the particle.

The equation of motion (Euler-Lagrange equation) for the Lagrangian given by (1) is

$$
m \ddot{q}+n \alpha q^{n-1}=0 .
$$

Instead to solve the equation of motion above, we will consider an equivalent equation. By multiplying it by $q$, and inserting the definition of the total energy of the particle given by (6), we obtain:

$$
q \ddot{q}-\frac{n}{2} \dot{q}^{2}+\frac{n E}{m}=0 .
$$

It is worth mentioning that, if some few identifications are made, (14) is the same differential equation describing the evolution of the scale factor in the standard FriedmanRobertson-Walker (FRW) cosmological model [10] (in this connection, see also [11]).

A first integral of (14) is

$$
\dot{q}^{2}=\frac{2 E}{m}\left(1-\frac{\alpha}{E} q^{n}\right) .
$$


It should be noted that the signals for the total energy $E$ and for the constant $\alpha$ must be properly chosen. So, if $E>0$ the constant $\alpha$ can have any signal, but if $E \leq 0$ we must have a negative $\alpha$. The integration of (15) is achieved with the introduction of an auxiliary variable defined by $u=(\alpha / E) q^{n}$. With the aid of this transformation the first integral is written as

$$
\frac{d u}{d t}=n B u^{1-1 / n}(1-u)^{1 / 2}
$$

where

$$
B=\sqrt{\frac{2 \alpha}{m}}\left(\frac{E}{\alpha}\right)^{1 / 2-1 / n} .
$$

At this stage we could integrate (16), as it was done in Sec. (II). Instead, on may consider the inverse problem, i.e., the solutions for a differential equation expressing the time as a function of the auxiliary variable $u(q)$. This inversion is readily done through (16), which provides the first derivative of $t(u)$ :

$$
\frac{d t}{d u}=\frac{1}{n B} u^{1 / n-1}(1-u)^{-1 / 2},
$$

whereas the second derivative may be put in the form

$$
u(1-u) \frac{d^{2} t}{d u^{2}}+[c-(a+1) u] \frac{d t}{d u}=0 .
$$

where $c=1-1 / n$ and $a=1 / 2-1 / n$. This is Gauss's hypergeometric differential equation. Its general solution consists of a linear combination of two linearly independent solutions [13]:

$$
t(u)=C_{1} F(a, 0 ; c ; u)+C_{2} u^{1-c} F(1+a-c, 1-c ; 2-c ; u),
$$

where $F$ is the hypergeometric function, and $C_{1}, C_{2}$ are the arbitrary constants of integration to be determined by the boundary conditions. It is a property of the hypergeometric function that if any of the first two parameters is zero, then the series terminates (i.e., $F(a, 0 ; c ; u)=1)$. It thus follows that the solution (19) reduces to

$$
t=C_{1}+C_{2}\left(\frac{\alpha}{E}\right)^{1 / n} q F\left(\frac{1}{2}, \frac{1}{n} ; \frac{1}{n}+1 ; \frac{\alpha}{E} q^{n}\right),
$$

where we have substituted the value of the parameters $a$ and $c$. The case $n=2$ gives

$$
q(t)=\sqrt{\frac{E}{\alpha}} \sin \omega\left(t-t_{0}\right),
$$

where the constant $\omega=1 / C_{2}$ and $t_{0}=C_{1}$ remain to be determined.

\section{PARAMETRIC SOLUTIONS AND PERIODICITY}

As we have seen, if $E \neq 0$, the general solution $t(q)$ cannot be inverted to obtain explicitly $q(t)$. In such cases, parametric solutions are usually more enlightening. Some simplicity is achieved when we replace the pair $(t, q) \rightarrow(\tau, Q)$, where the "conformal time" $\tau$ and the new coordinate $Q$ are defined by

$$
d t=q(\tau) d \tau
$$

and

$$
Q=q^{-\frac{n}{2}}
$$

Under the above transformations, the expressions for the Lagrangian and energy E, given by (1) and (2), become

$$
\begin{aligned}
& L=\frac{2 m}{n^{2}} Q^{-2} Q^{\prime 2}-\alpha Q^{-2}, \\
& E=\frac{2 m}{n^{2}} Q^{-2} Q^{\prime 2}+\alpha Q^{-2},
\end{aligned}
$$

where a prime denotes derivative with respect to $\tau$. As one may check, the Euler-Lagrange equations are now given by

$$
Q Q^{\prime \prime}-Q^{\prime 2}-\frac{\alpha n^{2}}{2 m}=0
$$

and inserting $Q^{\prime 2}$ from the energy conservation law, we obtain

$$
Q^{\prime \prime}-\left(\frac{n^{2} E}{2 m}\right) Q=0 .
$$

This is an interesting result. The above equation describes the motion of a classical particle under the action of a linear force. If $E<0(E>0)$ the force is of restoring (repulsive) type, while the motion of a free particle corresponds to $E=0$. The general solution of (27) is

$$
Q=\frac{Q_{0}}{\sqrt{\varepsilon}} \sin \sqrt{\varepsilon}(\omega \tau+\delta),
$$

where $\varepsilon=-2 E / m, \omega=n / 2$ is the frequency of the oscillator (anti-oscillator) and $Q_{0}, \delta$ are integration constants. Since the energy $E$ may be negative, the "phase" $\delta$ may assume complex values. The constant $Q_{0}$ can be determined using the energy equation written in terms of the conformal time. It turns out that $Q_{0}=\sqrt{-2 \alpha / m}$. Substituting this into (28), and returning to the original coordinate $q$, we have

$$
q(\tau)=(-m / 2 \alpha)^{1 / n}\left(\frac{\sin \sqrt{\varepsilon} \omega \tau}{\sqrt{\varepsilon}}\right)^{-2 / n}
$$

where we have put $\delta=0$ in (28).

\section{RELATIVISTIC HAMILTON-JACOBI APPROACH}

Let us now consider the relativistic treatment for the homogeneous potential. For a single-particle the relativistic Lagrangean is

$$
L=-m c^{2} \sqrt{1-\frac{\dot{q}^{2}}{c^{2}}}-\alpha q^{n}
$$


The Hamiltonian function for the particle is given by the general formula

$$
\mathcal{H}=\dot{q} \frac{\partial L}{\partial \dot{q}}-L=\sqrt{p^{2} c^{2}+m^{2} c^{4}}+\alpha q^{n},
$$

where $p$ is the canonical momentum. The Hamilton-Jacobi equation for $S$ is obtained by replacing in (31) $p$ by $\partial S / \partial q$ and $\mathcal{H}$ by $-(\partial S / \partial t)$ :

$$
c^{2}\left(\frac{\partial S}{\partial q}\right)^{2}-\left(\frac{\partial S}{\partial t}+\alpha q^{n}\right)^{2}+m^{2} c^{4}=0 .
$$

As before, a solution for (32) can be found separating the variables in the form

$$
S(q, E, t)=W(q, E)-E t,
$$

where the integration constant $E$ is again the total energy. With the above choice one finds from (32)

$$
S=\frac{1}{c} \int \sqrt{\left(E-\alpha q^{n}\right)^{2}-m^{2} c^{4}} d q-E t,
$$

which should be compared with its classical version (5). The second integration constant arises out from the transformation equation $\left(\beta=\frac{\partial S}{\partial E}\right)$ and we have finally

$$
t+\beta=\frac{1}{c} \int \frac{E-\alpha q^{n}}{\sqrt{\left(E-\alpha q^{n}\right)^{2}-m^{2} c^{4}}} d q .
$$

Without loss of generality we take $q(t=0)=0$, so that $\beta=0$ in (35). We next suppose that the potential is a symmetric function about the origin, that is, we consider only even values for $n$. Then the motion will be limited between $V(-A)$ and $V(A)$ where the amplitude is now given by $A=$ $\left[\left(E-m c^{2}\right) / \alpha\right]^{1 / n}$, and, from (35), the period $T$ is determined by

$$
T=\frac{4}{c} \int_{0}^{A} \frac{d q}{\sqrt{1-\left(\frac{m c^{2}}{E-\alpha q^{n}}\right)^{2}}} .
$$

Writing the total energy as $E=m c^{2}(1+\varepsilon)$, and considering that the potential energy is small compared to the rest mass energy $m c^{2}$, relativistic corrections to second order in the period $T$ are

$$
T=\frac{4}{c} \int_{0}^{A} \frac{d q}{\sqrt{2 \kappa\left(A^{n}-q^{n}\right)}}\left(1+\frac{3 \kappa}{4}\left(A^{n}-q^{n}\right)\right),
$$

where $\kappa=\alpha / m c^{2}$. Introducing the variable change $y=(q / A)^{n}$ the above integral can be rewritten as

$$
\begin{aligned}
T= & \frac{4}{c A^{\frac{n}{2}-1} n \sqrt{2 \kappa}} \int_{0}^{1} y^{-\left(\frac{n-1}{n}\right)}(1-y)^{-\left(\frac{1}{2}\right)} d y \\
& +\frac{3 \kappa A^{\frac{n+2}{2}}}{c n \sqrt{2 \kappa}} \int_{0}^{1} y^{-\left(\frac{n-1}{n}\right)}(1-y)^{\frac{1}{2}} d y
\end{aligned}
$$

whose values are tabulated in terms of the complete Beta function[9]. Substituting for $\mathrm{K}$ we write the period as

$$
T=2 \sqrt{\frac{2 m}{\alpha n^{2}}} A^{1-n / 2} B(1 / n, 1 / 2)\left[1+\frac{3}{8} \frac{\alpha A^{n}}{m c^{2}}\left(\frac{2 n}{n+2}\right)\right] .
$$

Observe that for $n=2$ (relativistic harmonic oscillator), the above equation reduces to

$$
T=2 \pi \sqrt{\frac{m}{2 \alpha}}\left[1+\frac{3}{8} \frac{\alpha A^{2}}{m c^{2}}\right],
$$

where the term $\left(3 \alpha A^{2}\right) / 8 m c^{2}$ is the first order relativistic correction earlier obtained by Synge[8] following a different approach (see Appendix).

The general form (39) is an interesting one. The term multiplying the square bracket is just the nonrelativistic period $T_{0}$ for the bounded homogeneous potential as given by (9). The remaining term represents the relativistic correction

$$
\frac{\Delta T}{T_{0}}=\frac{3}{8} \frac{\alpha A^{n}}{m c^{2}}\left(\frac{2 n}{n+2}\right),
$$

and, although it depends of $n$, we see that for $n \gg 1$ it saturates around $\left(3 \alpha A^{n}\right) / 4 m c^{2}$ which is twice the relativistic correction for the harmonic oscillator.

In conclusion, we have shown that the classical version of homogeneous potential problem can completely be solved by the HJ approach. In the relativistic case we not succeed in obtaining an analytic solution, but the earlier Lagrangian Synge's treatment for the harmonic oscillator is readily generalized using the HJ transformation. Finally, we stress that although important from their own right, such problems may also have interest for describing some excited states appearing in quantum chromodynamics which have been usually described by the relativistic oscillator[14]. The effective homogeneous potential also appears naturally in the cosmological framework. In this case, the values of the power $n$ is closely related to the nature of the cosmic fluid $[10,11]$. Its usefulness for providing a possible classification scheme for cosmological models has also been recently discussed [15].

\section{Acknowledgment}

This work was partially supported by CNPq and CAPES (Brazilian Research Agencies). JASL is also grateful to FAPESP No. 04/13668-0.

\section{APPENDIX: REMARKS ON SYNGE'S INTEGRAL}

The relativistic harmonic oscillator $(n=2)$ was long ago discussed by Synge [8]. In this appendix, we show how the exact Synge's integral form for the period of the motion can be generalized for a power-law index.

For an arbitrary $n$, the period is given by (see (36))

$$
T_{n}=\frac{4}{c} \int_{0}^{A} \frac{d q}{\sqrt{1-\left(\frac{m c^{2}}{E-\alpha q^{n}}\right)^{2}}} .
$$


Now, by writing $E=m c^{2}+\alpha A^{n}$ one finds

$$
T_{n}=2 \sqrt{\frac{2 m}{\alpha A^{n}}} \int_{0}^{A} \frac{\left[1+2 \chi^{2}\left(1-(q / A)^{n}\right)\right] d q}{\sqrt{1-(q / A)^{n}+\chi^{2}\left(1-(q / A)^{n}\right)^{2}}},
$$

where $\chi^{2}=\alpha A^{n} /\left(2 m c^{2}\right)$.

Introducing the variable $\varphi$ by $q=A \sin ^{2 / n} \varphi$, the above integral becomes

$$
T_{n}=4 \sqrt{\frac{2 m}{\alpha n^{2}}} A^{1-n / 2} \int_{0}^{\frac{\pi}{2}} \frac{\sin ^{2 / n-1} \varphi\left(1+2 \chi^{2} \cos ^{2} \varphi\right) d \varphi}{\sqrt{1+\chi^{2} \cos ^{2} \varphi}},
$$

and, for $n=2$ we have

$$
T_{2}=4 \sqrt{\frac{m}{2 \alpha}} \int_{0}^{\frac{\pi}{2}} \frac{\left(1+2 \chi^{2} \cos ^{2} \varphi\right) d \varphi}{\sqrt{1+\chi^{2} \cos ^{2} \varphi}}
$$

which is the Synge's integral with a slight different notation (our $\alpha=k^{2} / 2$, and $A=a$ in Synge's work[8]). Finally, by expanding (44) in powers of $\chi$, the relativistic correction for the period as given by (39) is recovered. For $n=2$ it reduces to Synge's expression.
[1] H. Goldstein, Classical Mechanics (Addison-Wesley Publ. Co., Reading, Mass. 1980).

[2] P. R. Holland, The Quantum Theory of Motion (Cambridge UP, Cambridge, 1995).

[3] B. Carter, Phys. Lett. A26 , 399 (1968); Phys. Rev. 174, 1559 (1968).

[4] L. D. Landau and E. M. Lifshitz, The Classical Theory of Fields (Pergamon Press, New York, 1975)

[5] W. H. C. Freire, V. B. Bezerra, and J. A. S. Lima, Gen. Rel. Grav. 33, 1407 (2001); R. C. Santos, J. A. S. Lima, and V. B. Bezerra, Gen. Rel. Grav. 33, 1969 (2002).

[6] G. del-Valle, I. Campos, and J. L. Jiménez, Eur. J. Phys. 17, 253 (1996); I. Campos, G. del-Valle, J. L. Jiménez, Eur. J. Phys. 24, 469 (2003).

[7] L. A. Pars, A Treatise on Analytical Dynamics (John Wiley \& Sons, New York, 1965).
[8] J. L. Synge, Classical Dynamics, in Encyclopedia of Physics, Vol. III, 210-214 (1960).

[9] M. Abramowitz and I. A. Stegun, Handbook of Mathematical Functions (Dover, New York, 1970).

[10] J. A. S. de Lima, J. A. M. Moreira, and J. Santos, Gen. Rel. Grav. 30, 425 (1998).

[11] M. Szydlowski and W. Czaja, Phys. Rev. D 69083518 (2004).

[12] M. J. D. Assad and J. A. S. Lima, Gen. Rel. Grav. 20, 427 (1988).

[13] J. B. Seaborn, Hypergeometric Functions and Their Applications (Springer-Verlag, New York, 1991).

[14] C. Dullemond and E. van Beveren, Phys. Rev. D28, 1028 (1983).

[15] M. Szydlowski and W. Czaja, Phys. Rev. D69 083507 (2004); M. Szydlowski and A. Kurek, gr-qc/0608098. 